\title{
Cardiac Remodeling in Obesity-Resistance Model is not Related to Collagen I and III Protein Expression
}

Scarlet Marques de Oliveira, ${ }^{1 \oplus}$ Jéssica Leite Garcia, ${ }^{\circledR}$ Danielle Fernandes Vileigas, ${ }^{1}$ Dijon Henrique Salomé de

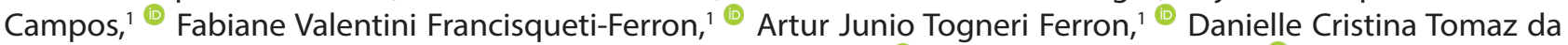
Silva-Bertani, ${ }^{1}$ Carlos Roberto Padovani, ${ }^{2}$ Camila Renata Corrêa, ${ }^{1}{ }^{\circledR}$ Antonio Carlos Cicogna' ${ }^{10}$

São Paulo State University (UNESP), Botucatu Medical School, Botucatu,' SP - Brazil

São Paulo State University (UNESP), Institute of Bioscience, ${ }^{2}$ Botucatu, SP - Brazil

\section{Abstract}

Background: As some individuals present resistance to obesity development, experiments have been trying to understand their susceptibility to cardiometabolic diseases.

Objetive: To evaluate if the cardiac remodeling was related to collagen protein expression change.

Methods: Male Wistar rats were randomized into two experimental groups: control diet (CD, n=15) or high-fat diet (HFD, $\mathrm{n}=15$ ) for 30 weeks. Rats fed with HFD were ranked based on their adiposity indexes and classified as obese $(\mathrm{Ob}, \mathrm{n}=8)$ or obesity-resistant $(\mathrm{ROb}, \mathrm{n}=6)$. Rats that failed to present the normal characteristic of the control group while fed with CD were excluded (Control, $\mathrm{n}=8$ ). Nutritional profile, comorbidities (dyslipidemia, hypertension, glucose metabolism, hyperleptinemia), cardiac remodeling, and collagen protein expression were evaluated. The groups were compared by One-Way ANOVA, together the Tukey post hoc test, with $\mathrm{p}<0.05$ considered significant.

Results: The $\mathrm{Ob}$ rats presented an increased adiposity index when compared to $\mathrm{C}$ and $\mathrm{ROb}$. Both groups $\mathrm{Ob}$ and $\mathrm{ROb}$ presented increased low-density lipoprotein (LDL), insulin, homeostatic model assessment of insulin resistance (HOMA- IR) and systolic blood pressure (SBP), and low high-density lipoprotein (HDL) levels when compared to the control group. The levels of triglycerides, non-esterified fatty acid (NEFA), and leptin were lower in ROb as compared to $\mathrm{Ob}$, but higher than the control group. The $\mathrm{Ob}$ and $\mathrm{ROb}$ groups presented cardiac remodeling, evidenced by echocardiographic and post-mortem analysis. The collagen protein expression did not differ among the groups.

Conclusion: The $\mathrm{ROb}$ animals present cardiac remodeling that is not related to collagen type I and III protein expression change.

Keywords: Obesity; Adiposity; Diet.

\section{Introduction}

Obesity is a chronic metabolic disease characterized by an excessive adipose tissue accumulation. ${ }^{1}$ It is considered a global epidemic and a major public health problem, ${ }^{2}$ since this disease can lead to nutritional, metabolic, hormonal, and cardiovascular changes, increasing the population's morbidity and mortality, and reducing life expectancy. ${ }^{3-5}$
The literature reports that the cause of main obesity, currently, is the inadequate dietary habits, with increased carbohydrate and/or fat consumption. 6,7 However, some individuals seem to present resistance to the development of obesity. Within this context, experiments using animals fed with a high-fat diet try to understand the pathophysiological susceptibility of obesity-resistant individuals to cardiometabolic diseases. ${ }^{8-10}$ 
Cardiac remodeling is well established in obesity conditions, since research has shown the relationship between time of obesity and myocardial collagen type I and III expression. ${ }^{11}$ However, no studies have evaluated the contribution of collagen expression to cardiac remodeling in obesity-resistant animal models, and the few studies that evaluated the cardiac changes in this condition found divergent results. Sá et al. ${ }^{9}$ found isolated papillary muscle contraction impairment, while Carroll et al. ${ }^{10}$ found cardiac no abnormalities in obesity-resistant animals fed with a high-fat diet.

Considering this situation, the primary aim of this study was to verify the presence of cardiac remodeling in obesity-resistant animals fed with a high-fat diet. An additional aim was to evaluate if the cardiac remodeling was related to collagen I and III protein expression change.

\section{Methods}

\section{Animals and experimental protocol}

Male Wistar rats (60-day-old) were randomly divided into two experimental groups to receive a control diet $(\mathrm{CD}, \mathrm{n}=15)$ or a high-fat diet (HFD, $\mathrm{n}=15)$ for 30 weeks. The sample size and the experimental period were based on previous studies conducted by our research group. ${ }^{9,11,12}$ Animals were kept in individual cages with controlled temperature $\left(24 \pm 2{ }^{\circ} \mathrm{C}\right)$, humidity $(55 \pm 5 \%)$, and light (12-h light/dark cycle). The diet and water were ad libitum. The experimental procedures were performed according to the "Guide for the Care and Use of Laboratory Animals"9,13 and approved by the Animal Ethics Committee of the Botucatu Medical School (991/2012). At the end of the experimental protocol, after $8 \mathrm{~h}$ fasting, the animals were euthanized by decapitation after intraperitoneal anesthesia with a mixture of ketamine $(1 \mathrm{mg} / \mathrm{kg})$ and xylazine $(100 \mathrm{mg} / \mathrm{kg}$ ) (Syntec, Rhobifarma Indústria Farmacêutica Ltda., Hortolândia, São Paulo, Brazil). The blood and the cardiac samples were collected and stored at $-20^{\circ} \mathrm{C}$ for further analysis.

\section{Diet composition}

The diets used in this study have been described elsewhere ${ }^{11,12,14,15}$ and the composition followed AIN93 recommendations, consisting of the following ingredients: corn bran, soybean hull, soybean bran, dextrin, salt, vitamin and mineral complex, palm kernel oil, and soybean oil.
The CD contained $31.0 \%$ of $\mathrm{kcal}$ from protein, $51.6 \%$ from carbohydrates, and $17.4 \%$ from fat. The HFD contained $18.7 \%$ of $\mathrm{kcal}$ from protein, $41.6 \%$ from carbohydrates, and $39.7 \%$ from fat. The content of saturated/unsaturated fatty acids was $61.5 \% / 38.5 \%$ in CD and $64.8 \% / 35.2 \%$ in HFD. The energetic densities from the diets were: HFD = $3.85 \mathrm{kcal} / \mathrm{g}$ and $\mathrm{CD}=3.10 \mathrm{kcal} / \mathrm{g}$.

\section{Nutritional profile}

The nutritional profile was determined by food and calorie intake, feed efficiency, final body weight, and adiposity index. Dietary intake and body weight were measured weekly. The calorie intake was determined by the following formula: weekly food intake multiplied by the energy value of each diet $(\mathrm{g} \times \mathrm{kcal})$. To analyze the animal's capacity to convert the consumed food energy in body weight, feed efficiency was calculated by dividing the total body weight gain (g) by the total energy intake (kcal). The total body fat was obtained by the sum of epididymal, retroperitoneal, and visceral deposits. The adiposity index was calculated by the total body fat divided by the final body weight and multiplied by $100.15,16$

\section{Determination of Obesity and Obesity Resistance}

A criterion based on the adiposity index was used to determine the occurrence of obesity and obesity resistance according to several authors. ${ }^{10,17-19}$ After 30 weeks, the rats that consumed HFD were ranked based on their adiposity indexes. Therefore, the animals that received HFD and presented the highest adiposity indexes were classified as obese $(\mathrm{Ob}, \mathrm{n}=8)$; the animals that consumed a high-fat diet and presented adiposity indexes similar to control animals were classified as obesity-resistant ( $\mathrm{ROb}, \mathrm{n}=6$ ). Rats that failed to present the normal characteristic of the control group, while fed with a standard diet, were excluded $(n=8)$.

\section{Metabolic and hormonal evaluation}

The metabolic evaluation included plasma lipid and glucose levels, as well as insulin resistance, whereas the hormonal evaluation was assessed by the concentrations of leptin and insulin.

The triglycerides, total cholesterol (TC), high- and low-density lipoprotein (HDL and LDL) levels were determined using a specific kit (BIOCLIN $®$, Belo Horizonte, MG, Brazil) and analyzed by the automated 
enzymatic colorimetric method (Chemistry Analyzer BS-200, Mindray Medical International Limited, Shenzhen, China). The non-esterified fatty acid (NEFA) concentrations were evaluated by colorimetric kit (WAKO Pure Chemical Industries Ltd., Osaka, Japan).

Glycemia was analyzed in blood samples collected from the tails of the animals, using a handheld glucometer (Accu-Chek Go Kit, Roche Diagnostic Brazil Ltda, São Paulo, SP, Brazil). The homeostatic model assessment of insulin resistance (HOMA-IR) was used as an insulin resistance index, calculated according to the formula: HOMA-IR $=[$ fasting glucose $(\mathrm{mmol} / \mathrm{L}) \times$ fasting insulin $(\mu \mathrm{U} / \mathrm{mL})] / 22.5^{7}$.

The insulin and leptin levels were analyzed by the enzyme-linked immunosorbent assay (ELISA) method (EMD Millipore Corporation, Billerica, MA, USA). The reading was performed using a microplate reader (Spectra MAX 190, Molecular Devices, Sunnyvale, CA, USA).

\section{Echocardiographic Study}

The analysis was performed with live animals by transthoracic echocardiography, using a Vivid S6 system equipped with a multifrequency ultrasonic transducer 5.0 to $11.5 \mathrm{MHz}$ (General Electric Medical Systems, Tirat Carmel, Israel). The animals were lightly anesthetized by intraperitoneal injection with a mixture of ketamine $(50 \mathrm{mg} / \mathrm{kg})$ and xylazine $(1 \mathrm{mg} / \mathrm{kg})$ and put in left decubitus position. Only one examiner performed all of the exams. The heart image structural measurements were obtained in one-dimensional mode (M-mode) guided by the images in two-dimensional mode with the transducer in the parasternal position, minor axis. Left ventricular (LV) evaluation was performed with the cursor M-mode just below the mitral valve plane at the level of the papillary muscles. The echocardiographic analysis was performed according to that established in prior studies. ${ }^{19-21}$

\section{Morphometric variables}

- Maximum left atrium diameter (LA, cm);

- Left ventricular diastolic and systolic diameters of the left ventricle (LV, mm): LVDD and LVSD, respectively;

- Interventricular septum diastolic thickness (IVSDT) and posterior wall diastolic thickness (PWDT) of the LV (mm): IVSDT and PWDT, respectively;

- Relative thickness of the LV $($ LVRT $)=(2 \times$ PWDT $) /$ LVDD;
- Left ventricular mass $(\mathrm{LVM}, \mathrm{g})=0.8 \times\{1.04 \times[($ IVSDT + PWDT + LVDD) $)^{3}-$ LVDD $\left.\left.^{3}\right]\right\}+0.6$;

- LVM index (LVMI, g/m $\mathrm{m}^{2.7}$ )= LVM/Height ${ }^{2.7}$ where LVMI is LV mass indexed to height.

\section{Systolic function variables}

- Cardiac output (CO) was calculated by multiplying the systolic volume by the heart rate.

\section{Diastolic function variables}

- Maximum early ventricular filling velocity (E wave peak, cm/s): obtained by spectral Doppler recording of the transmitral diastolic flow;

- Maximum late filling velocity during atrial contraction (A wave peak, cm/s): obtained by spectral Doppler recording of the transmitral diastolic flow;

- E-wave deceleration time (ms) corresponding to the time between the initial velocity peak of the mitral transvalvular flow and its extrapolation to the baseline.

\section{Systolic blood pressure}

The Systolic blood pressure (SBP) was measured by tail plethysmography, using a Narco Bio-System ${ }^{\circledR}$ ElectroSphygmomanometer, model 709-0610 (International Biomedical, Austin, TX, USA). The animals were warmed in a wooden box $(50 \times 40 \mathrm{~cm})$ between $38-40^{\circ} \mathrm{C}$ for $4-5 \mathrm{~min}$ to stimulate arterial vasodilation. After this procedure, a cuff with a sensor was placed in the proximal region of the tail, coupled to the electro-sphygmomanometer. The cuff was inflated to $200 \mathrm{mmHg}$ pressure and subsequently deflated. ${ }^{15,16}$ The arterial pulsations were recorded in a computerized data acquisition system (AcqKnowledge ${ }^{\circledR}$ MP100, Biopac Systems Inc., Santa Barbara, CA). The average of two readings was recorded for each measurement.

\section{Post-Death Morphological Analysis}

After euthanasia, the animals were submitted to thoracotomy, and the hearts, ventricles, and tibia were separated, dissected, weighed, and measured. Cardiac remodeling was determined by analyzing the weight of the heart and the left (LV) and right (RV) ventricles, and their correlation with the tibial length.

\section{Myocardial collagen types I and III protein expression}

The Western Blot analysis was performed to evaluate the types I and III collagen protein expression. Briefly, 
the LV samples were rapidly frozen in liquid nitrogen and subsequently homogenized in a solution containing RIPA buffer (Amresco LLC, Solon, OH, USA), together with protease (Sigma-Aldrich, St. Louis, MO) and phosphatase (Roche Diagnostics, Indianapolis, IN, USA) inhibitors. The samples were subjected to SDS-PAGE in $10 \%$ polyacrylamide gel and were then electrotransferred to a nitrocellulose membrane (Amersham Biosciences, Piscataway, NJ, USA). The blotted membrane was blocked (5\% nonfat dry milk, $20 \mathrm{mmol} / \mathrm{L}$ Tris- $\mathrm{HCl}$ $\mathrm{pH} 7.4,137 \mathrm{mmol} / \mathrm{L} \mathrm{NaCl}$ and $1 \%$ Tween 20 ) for $2 \mathrm{~h}$ at room temperature and then incubated overnight at $4-8^{\circ} \mathrm{C}$ with primary antibody against collagen type I (1:10000) and collagen type III (1:10000) (Abcam, Cambridge, MA, USA). The immunoblots were washed three times with TBS-T and incubated for $1.5 \mathrm{~h}$ with peroxidase-conjugated anti-rabbit secondary antibody (1:2000) (Abcam, Cambridge, MA, USA), and then washed again three times with TBS-T and incubated with ECL (Enhanced Chemi-Luminescence, Amersham Biosciences, Piscataway, NJ, USA) for chemiluminescence detection. Blots were analyzed on Scion Image software (Scion Corporation, Frederick, $\mathrm{MD}, \mathrm{USA}$ ) and protein expressions were normalized to $\beta$-actin expression (1:1000) (Santa Cruz Biotechnology, Santa Cruz, CA, USA).

\section{Statistical analysis}

The data were submitted to Kolmogorov-Smirnov normality test. Parametric variables were compared by One-Way analysis of variance (ANOVA) and complemented with the Tukey post hoc test for multiple comparisons when significant differences were found $(\mathrm{p}<$ 0.05 ). All the results are presented as mean \pm standard deviation. Statistical analyses were performed using Sigma Stat for Windows Version 3.5. (Systat Software, Inc., San Jose, CA, USA). The level of significance considered was $5 \%$.

\section{Results}

Table 1 presents the nutritional profile of the groups. It is possible to verify that both $\mathrm{Ob}$ and $\mathrm{ROb}$ groups presented lower food intake compared to the control group. Feed efficiency, final body weight, weight gain, and adiposity index were higher in the Ob group when compared to the control group. The ROb presented only a reduced adiposity index when compared to the $\mathrm{Ob}$ group, with no difference in the other variables.

The metabolic, cardiovascular, and hormonal parameters are presented in Table 2. Both groups, $\mathrm{Ob}$ and Rob, presented increased LDL, insulin, HOMA- IR and $\mathrm{SBP}$, and low HDL levels when compared to the control group. The triglycerides, NEFA, and leptin levels were lower in the Rob group when compared to the Ob group, but higher than the control group.

The echocardiographic parameters are presented in the Figure 1. The $\mathrm{Ob}$ and $\mathrm{ROb}$ groups presented cardiac remodeling, characterized by increased LVDD, LVSD, left atrium, and estimated LV mass when compared to the control group. The systolic dysfunction, characterized by reduced cardiac output, was detected in both the $\mathrm{Ob}$ and

Table 1 - Nutritional profile

\begin{tabular}{|c|c|c|c|}
\hline Variables & $C(n=8)$ & $\mathrm{Ob}(n=8)$ & $\mathrm{ROb}(\mathrm{n}=6)$ \\
\hline Food intake (g/day) & $25.1 \pm 2.3$ & $21.1 \pm 1.8^{*}$ & $20.2 \pm 1.4^{*}$ \\
\hline Calorie intake (kcal/day) & $74.2 \pm 7.1$ & $76.9 \pm 6.7$ & $73.9 \pm 5.1$ \\
\hline Feed efficiency (\%) & $1.45 \pm 0.08$ & $1.82 \pm 0.20^{*}$ & $1.64 \pm 0.14$ \\
\hline Final body weight (g) & $506 \pm 46.9$ & $592 \pm 60.2^{*}$ & $546 \pm 36.4$ \\
\hline Weight gain (g) & $226 \pm 21,3$ & $296 \pm 51,2^{*}$ & $255 \pm 24,3$ \\
\hline Adiposity index (\%) & $5.91 \pm 0.59$ & $10.21 \pm 1.41^{*}$ & $6.31 \pm 0.51 \#$ \\
\hline
\end{tabular}


Table 2 - Metabolic, cardiovascular, and hormonal parameters

\begin{tabular}{|c|c|c|c|}
\hline Variables & $C(n=8)$ & $\mathrm{Ob}(\mathrm{n}=8)$ & $\mathrm{ROb}(\mathrm{n}=6)$ \\
\hline Triglycerides (mg/dL) & $50.1 \pm 10.3$ & $99.6 \pm 19.8^{*}$ & $73.1 \pm 10.6^{* \#}$ \\
\hline NEFA (mmol/L) & $0.345 \pm 0.033$ & $0.642 \pm 0.037^{*}$ & $0.462 \pm 0.034^{*} \#$ \\
\hline HDL (mg/dL) & $28.7 \pm 2.2$ & $22.1 \pm 2.6^{*}$ & $22.3 \pm 2.9^{*}$ \\
\hline LDL (mg/dL) & $19.9 \pm 2.1$ & $33.4 \pm 5.1^{*}$ & $30.9 \pm 3.7^{*}$ \\
\hline Glucose (mg/dL) & $117 \pm 13$ & $169 \pm 13^{*}$ & $136 \pm 17 \#$ \\
\hline Insulin (ng/mL) & $3.39 \pm 0.49$ & $9.18 \pm 2.14^{*}$ & $7.91 \pm 2.12^{*}$ \\
\hline HOMA-IR & $18.6 \pm 4.5$ & $75.1 \pm 14.2^{*}$ & $21.1 \pm 8.6^{*}$ \\
\hline Leptin (ng/mL) & $4.23 \pm 1.11$ & $27.34 \pm 3.36^{*}$ & $10.76 \pm 3.21^{*} \#$ \\
\hline $\mathrm{SBP}(\mathrm{mmHg})$ & $116 \pm 7$ & $135 \pm 3^{*}$ & $133 \pm 12^{*}$ \\
\hline \multicolumn{4}{|c|}{$\begin{array}{l}\text { Data presented as mean } \pm \text { standard deviation. } n \text { : Number of animals; } C \text { : control; Ob: obese; ROb: obesity-resistant. TC: total cholesterol; HDL: high- } \\
\text { density lipoprotein; LDL: low-density lipoprotein; NEFA: non-esterified fatty acids; HOMA-IR: Homeostatic Model Assessment - Insulin Resistance; } \\
\text { SBP: Systolic blood pressure. *versus C; } p<0.05 \text {; \#versus Ob, } p<0.05 \text {; One-way ANOVA for independent samples and Tukey's post hoc test. }\end{array}$} \\
\hline
\end{tabular}

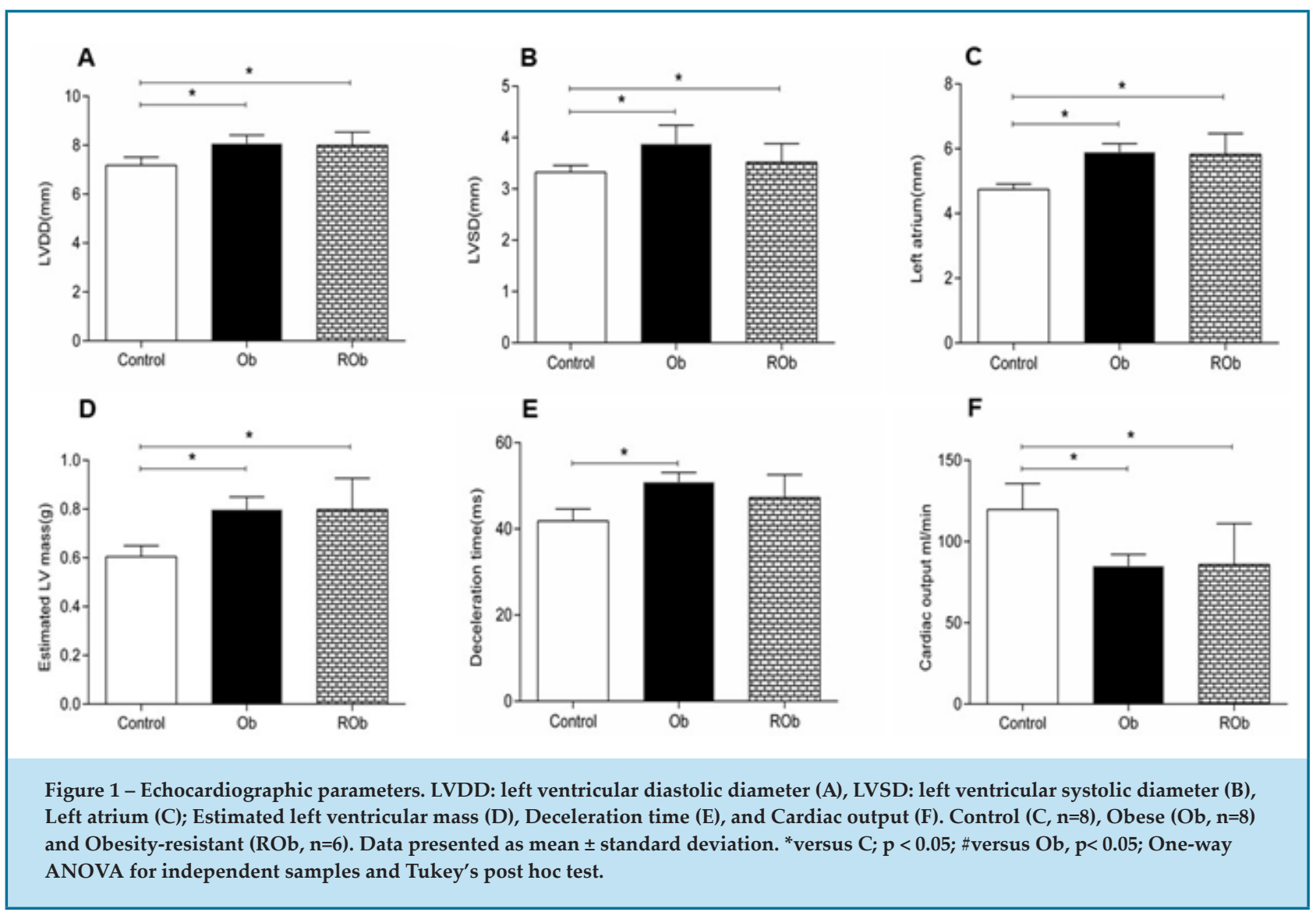


ROb groups. The diastolic dysfunction appeared only in the $\mathrm{Ob}$ group (increased deceleration time).

Cardiac remodeling was also confirmed in both the $\mathrm{Ob}$ and $\mathrm{ROb}$ groups by the cardiac post-mortem analysis, as these animals presented higher heart weight, LV weight, RV weight, heart/ tibia length, LV/ tibia length, and RV/ tibia length when compared to the control group (Figure 2).

Figure 3 shows the collagen type I (figure 3A) and the collagen type III (figure $3 \mathrm{~B}$ ) protein expression. No difference was found among the groups.

\section{Discussion}

Since obesity and its related disorders are becoming increasingly prevalent, several researchers have been using high-fat diet models to induce obesity, typically characterized by weight gain and increased body fat. ${ }^{7,15,22,23}$ In the present study, we chose $\sim 40 \%$ of $\mathrm{kcal}$ from fat, as we believe this is closer to what is consumed by humans. However, some individuals remain resistant to becoming obese, a condition also observed in some animals' fed with high-fat diets, which are defined as obesity-resistant animals. ${ }^{8-10,24,25}$ Within this context, some authors have reported that around $40 \%$ of the animals fed with a high-fat diet are classified as ROb. ${ }^{8-10,23}$ Some possible pathways to explain the obesity resistance include: increased expression of some thermogenic enzymes and decreased expression of lipogenic enzymes in adipose tissues of $\mathrm{ROb}$ rats, as well as the suppression of lipogenesis and the acceleration of fatty-acid oxidation in visceral fat. ${ }^{8}$

Several experiments have demonstrated that obese rats due to a high-fat diet intake develop obesity-related disorders that are similar to human disorders, such as glucose intolerance, insulin resistance, hypertension, and dyslipidemia. ${ }^{16,22,26}$ However, in ROb models, there are controversies regarding the presence of comorbidities..$^{9,10,23,27}$ In the current study, the $\mathrm{ROb}$ group presented relevant metabolic, hormonal, and cardiovascular changes commonly found in obesity and associated with increased adiposity.$^{28} \mathrm{As}$ the $\mathrm{ROb}$ group presented a weight gain and an adiposity index similar to the $\mathrm{C}$ group, it demonstrates that all the disorders were independent of adiposity gain. According to the literature, the intake of processed foods rich in fats, especially saturated fat, is one of the main causes for
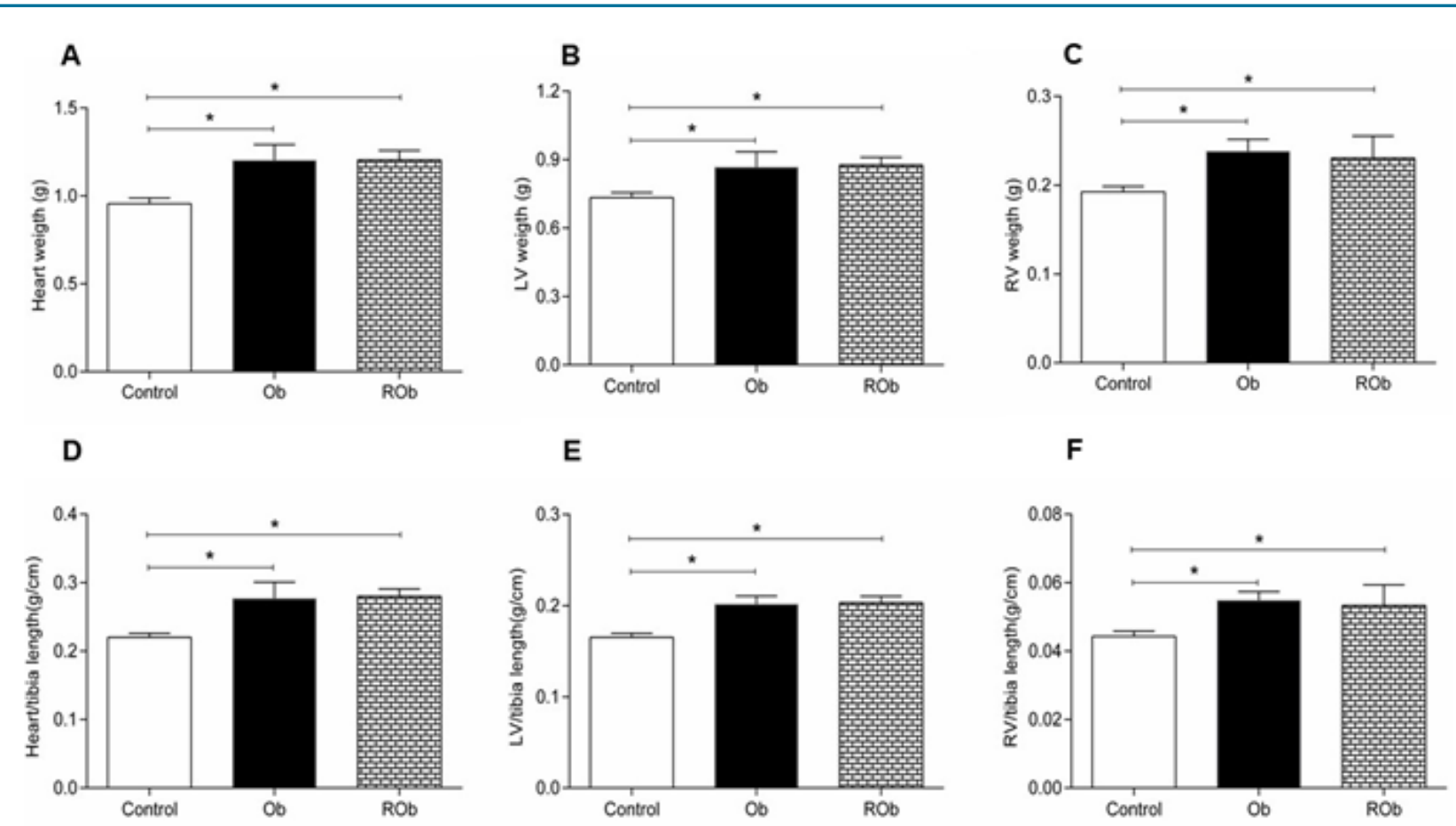

Figure 2 - Cardiac remodeling. Heart weight (A), LV: left ventricle weight (B), RV: right ventricle weight (C); Heart/tibia length (D), LV/ tibia length (E), and RV/tibia length (F). Control $(C, n=8)$, Obese $(O b, n=8)$, and Obesity-resistant (ROb, $n=6)$. Data presented as mean \pm standard deviation. *versus $\mathrm{C}$; $\mathrm{p}<0.05$; \#versus $\mathrm{Ob}, \mathrm{p}<0.05$; One-way ANOVA for independent samples and Tukey's post hoc test. 

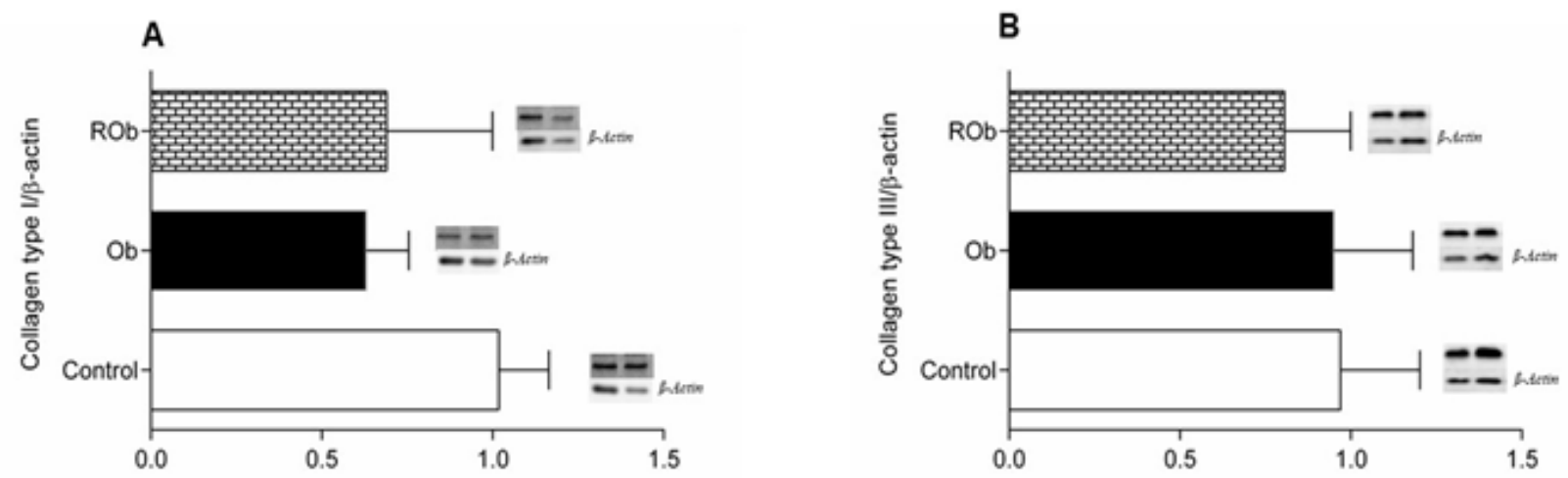

Figure 3 - Western blot analysis of collagen type I (A) and type III (B) in the hearts of the control $(C, n=4)$ and obese I (Ob, n=4) and II $(\mathrm{ROb}, \mathrm{n}=4)$ rats. Western blot bands were normalized by $\beta$-actin. Data presented as mean \pm standard deviation. $\mathrm{n}$ : Number of animals; C: control; Ob: obese; ROb: obesity-resistant. *versus C; $\mathrm{p}<0.05$; \#versus $\mathrm{Ob}, \mathrm{p}<0.05$; One-way ANOVA for independent samples and Tukey's post hoc test.

obesity and is considered an isolated cause of metabolic disorder development due the pro-inflammatory effect of this nutrient. ${ }^{29}$ Corroborating this finding, the $\mathrm{ROb}$ group presented increased an HOMA-IR index when compared to the control group, indicating impairment in carbohydrate metabolism as well as dyslipidemia, characterized by increased triglycerides and LDL, and reduced HDL.

Increased leptin and insulin are common in obesity. ${ }^{16,22}$ However, the present study also observed this condition in the $\mathrm{ROb}$ animals. Increased insulin can be due to insulin resistance or elevated gastric inhibitory polypeptide levels induced by high-saturated fatty acid intake. ${ }^{30}$ At the same time, hyperinsulinemia stimulates and increases the leptin secretion by adipose tissue through the PI3K/Akt/ mTOR pathway, which can explain the increased leptin levels in the $\mathrm{ROb}$ animals that did not present increased body fat. ${ }^{31}$

The metabolic responses to hyperinsulinemia and hyperleptinemia are well established in the literature. ${ }^{22,26}$ Nevertheless, these conditions also promote responses in other target organs, such as the heart. ${ }^{16,32}$ In obesity, the high hormone levels trigger hypertrophic responses in the heart by activating specific signaling pathways. ${ }^{33-36}$ However, in obesity-resistance models, the establishment of cardiac remodeling seems controversial.

Our results confirm the primary aim of this experiment, since the presence of cardiac remodeling in the $\mathrm{ROb}$ animals was verified by both echocardiographic and morphological post-mortem analysis,. The majority of cardiac diseases are followed by heart mass and morphologic changes. Due to the cardiac cell's incapacity to divide into the adult phase, the remodeling process usually occurs because of cardiomyocytes hypertrophy in response to a hemodynamic overload. ${ }^{37}$

Hemodynamic and hormonal changes promote extracellular matrix remodeling, altering its gene expression. ${ }^{38,39}$ There are two main types of collagen in the heart, types I and III, which are responsible by cardiac rigidity. ${ }^{40}$ However, different obesity models have demonstrated controversial results about collagen synthesis and degradation in the heart. ${ }^{38,39,41}$ In this sense, this study had as secondary aim to evaluate if the cardiac remodeling was related to collagen I and III protein expression changes. Our results showed that no difference was found in the collagen protein expression among the groups. Thus, other pathways that influence cardiac remodeling and should be addressed in future studies include insulin/ PI3k/Akt/ PKB, ${ }^{33,37}$ leptin/ RhoA/ ROCK/ p38, ${ }^{34,35}$ oxidative stress, ${ }^{42}$ and inflammation. ${ }^{43}$

\section{Study Limitations}

Limitations of this study include the absence of histological analysis for collagen evaluation and the small sample size.

\section{Conclusion}

Considering the results presented in this study, it is possible to conclude that obesity-resistant animals present cardiac remodeling that is not related to collagen type I and III protein expressions. 


\section{Author contributions}

Conception and design of the research: Oliveira SM and Cicogna AC. Acquisition of data: Oliveira SM, Campos DHS, Silva-Bertani DCT and Vileigas DF. Analysis and interpretation of the data: Oliveira SM, Vileigas DF, Ferron AJT, Silva-Bertani DCT and Corrêa CR. Statistical analysis: Francisqueti-Ferron FV, Ferron AJT and Padovani CR. Obtaining financing: Oliveira SM and Cicogna AC. Writing of the manuscript: Oliveira SM, Garcia JL, FrancisquetiFerron FV, Ferron AJT and Cicogna AC. Critical revision of the manuscript for intellectual content: Ferron AJT, Corrêa $\mathrm{CR}$ and Cicogna AC.

\section{Potential Conflict of Interest}

No potential conflict of interest relevant to this article was reported.

\section{References}

1. World Health Organization. [Internet]. Obesity and overweight. Geneva: World Health Organization; 2020. [cited 2021 Jun 02]. Available from: https:// www.who.int/news-room/fact-sheets/detail/obesity-and-overweight

2. NCD Risk Factor Collaboration (NCD-RisC). Worldwide trends in bodymass index, underweight, overweight, and obesity from 1975 to 2016: a pooled analysis of 2416 population-based measurement studies in 128.9 million children, adolescents, and adults. Lancet. 2017;390(10113):262742. doi: 10.1016/S0140-6736(17)32129-3

3. Popkin BM. Relationship between shifts in food system dynamics and acceleration of the global nutrition transition. Nutr Rev. 2017;75(2):73-82. doi: 10.1093/nutrit/nuw064

4. Elagizi A, Kachur S, Lavie CJ, Carbone S, Pandey A, Ortega FB, Milani RV. An Overview and Update on Obesity and the Obesity Paradox in Cardiovascular Diseases. Prog Cardiovasc Dis. 2018;61(2):142-50. doi: 10.1016/j.pcad.2018.07.003

5. Popkin BM, Adair LS, Ng SW. Global nutrition transition and the pandemic of obesity in developing countries. Nutr Rev. 2012;70(1):3-21. doi: 10.1111/j.1753-4887.2011.00456.x.

6. Francisqueti FV, Minatel IO, Ferron AJT, Bazan SGZ, Silva VDS, Garcia JL, et al. Effect of gamma-oryzanol as therapeutic agent to prevent cardiorenal metabolic syndrome in animals submitted to high sugar-fat diet. Nutrients. 2017;9(12):1299. doi: 10.3390/nu9121299.

7. Ferron AJT, Aldini G, Francisqueti-Ferron FV, Silva CCVA, Bazan SGZ, Garcia JL, et al. Protective effect of tomato-oleoresin supplementation on oxidative injury recoveries cardiac function by improving $\beta$-adrenergic response in a diet-obesity induced model. Antioxidants. 2019;8(9):368. doi: 10.3390/antiox8090368

8. Akieda-Asai S, Koda S, Sugiyama M, Hasegawa K, Furuya M, Miyazato $\mathrm{M}$, et al. Metabolic features of rats resistant to a high-fat diet. Obes Res Clin Pract. 2013;7(4):e243-50. doi: 10.1016/j.orcp.2013.01.004.

9. Sá FG, Lima-Leopoldo AP, Jacobsen BB, Ferron AJ, Estevam WM, Campos $\mathrm{DH}$, et al. Obesity resistance promotes mild contractile dysfunction associated with intracellular $\mathrm{Ca} 2+$ handling. Arq Bras Cardiol. 2015;105(6):588-96. doi: 10.5935/abc.20150134.

10. Carroll JF, Zenebe WJ, Strange TB. Cardiovascular function in a rat model of diet-induced obesity. Hypertension. 2006;48(1):65-72. doi: 10.1161/01. HYP.0000224147.01024.77.

\section{Sources of Funding}

This study was funded by Fundação de Amparo à Pesquisa do Estado de São Paulo - FAPESP (grants: 2013/23954-0 and 2012/21024-2).

\section{Study Association}

This study is not associated with any thesis or dissertation work.

\section{Ethics approval and consent to participate}

This study was approved by the Ethics Committee on Animal Experiments of the FMB-UNESP under the protocol number 991/2012.

11. Silva DC, Lima-Leopoldo AP, Leopoldo AS, Campos DH, Nascimento AF, Oliveira SA Jr, et al. Influence of term of exposure to high-fat dietinduced obesity on myocardial collagen type I and III. Arq Bras Cardiol. 2014;102(2):157-63. doi: 10.5935/abc.20130232

12. Silva-Bertani DCTD, Vileigas DF, Mota GAF, Souza SLB, Tomas LC, Campos DHS, et al. Decreased collagen type i is associated with increased metalloproteinase-2 activity and protein expression of leptin in the myocardium of obese rats. Arq Bras Cardiol. 2020;115(1):61-70. doi: 10.36660/abc.20180143.

13. Canadian Council on Animal Care. Conseil canadien de protection des animaux. Guide to the Care and Use of Experimental Animals. 2nd ed. Ottawa: CCAC; 2020.

14. Adorni CS, Corrêa CR, Vileigas DF, Campos DHS, Padovani CR, Minatel $\mathrm{IO}$, et al. The influence of obesity by a diet high in saturated fats and carbohydrates balance in the manifestation of systemic complications and comorbidities. Nutrire. 2017;42:16. doi: 10.1186/s41110-017-0042-1.

15. Vileigas DF, Deus AF, Silva DC, Tomasi LC, Campos DH, Adorni CS, et al. Saturated high-fat diet-induced obesity increases adenylate cyclase of myocardial $\beta$-adrenergic system and does not compromise cardiac function. Physiol Rep. 2016;4(17):e12914. doi: 10.14814/phy2.12914.

16. Ferron AJ, Jacobsen BB, Sant'Ana PG, Campos DH, Tomasi LC, Luvizotto RA, et al. Cardiac dysfunction induced by obesity is not related to $\beta$-adrenergic system impairment at the receptor-signalling pathway. PLoS One. 2015;10(9):e0138605. doi: 10.1371/journal.pone.0138605.

17. Boustany-Kari CM, Gong M, Akers WS, Guo Z, Cassis LA. Enhanced vascular contractility and diminished coronary artery flow in rats made hypertensive from diet-induced obesity. Int J Obes. 2007;31(11):1652-9. doi: $10.1038 /$ sj.ijo.0803426.

18. Levin BE, Keesey RE. Defense of differing body weight set points in diet-induced obese and resistant rats. Am J Physiol. 1998;274(2):412-9. doi: 10.1152/ajpregu.1998.274.2.R412

19. Rodrigues JCS, Luvizutto GJ, Costa RDM, Prudente RA, Silva TR, Souza JT, et al. Influence of an exercise program on cardiac remodeling and functional capacity in patients with stroke (CRONuS trial): study protocol for a randomized controlled trial. Trials. 2019;20(1):298. doi: 10.1186/ s13063-019-3328-1. 
20. Lang RM, Bierig M, Devereux RB, Flachskampf FA, Foster E, Pellikka $\mathrm{PA}$, et al. Recommendations for chamber quantification: a report from the American Society of Echocardiography's Guidelines and Standards Committee and the Chamber Quantification Writing Group, developed in conjunction with the European Association of Echocardiography, a branch of the European Society of Cardiology. J Am Soc Echocardiogr. 2005;18(12):1440-63. doi: 10.1016/j.echo.2005.10.005.

21. Nagueh SF, Appleton CP, Gillebert TC, Marino PN, Oh JK, Smiseth OA, et al. Recommendations for the evaluation of left ventricular diastolic function by echocardiography. J Am Soc Echocardiogr. 2009;22(2):107-33. doi: 10.1016/j.echo.2008.11.023

22. Francisqueti FV, Ferron AJT, Hasimoto FK, Alves PHR, Garcia JL, Santos $\mathrm{KC}$, et al. Gamma oryzanol treats obesity-induced kidney injuries by modulating the adiponectin receptor 2/PPAR- $\alpha$ axis. Oxid Med Cell Longev. 2018;2018:1278392. doi: 10.1155/2018/1278392.

23. Oliveira SA Jr, Dal Pai-Silva M, Martinez PF, Campos DH, LimaLeopoldo AP, Leopoldo AS, et al. Differential nutritional, endocrine, and cardiovascular effects in obesity-prone and obesity-resistant rats fed standard and hypercaloric diets. Med Sci Monit. 2010;16(7):BR208-17.

24. Madsen AN, Hansen G, Paulsen SJ, Lykkegaard K, Tang-Christensen M, Hansen HS, et al. Long-term characterization of the diet-induced obese and diet-resistant rat model: a polygenetic rat model mimicking the human obesity syndrome. J Endocrinol. 2010;206(3):287-96. doi: 10.1677/ JOE-10-0004.

25. Nascimento AP, Monte-Alto-Costa A. Both obesity-prone and obesityresistant rats present delayed cutaneous wound healing. Br J Nutr. 2011;106(4):603-11. doi: 10.1017/S0007114511000468.

26. Ferron AJT, Francisqueti FV, Minatel IO, Silva CCVA, Bazan SGZ, Kitawara $\mathrm{KAH}$, et al. Association between cardiac remodeling and metabolic alteration in an experimental model of obesity induced by western diet. Nutrients. 2018;10(11):1675. doi: 10.3390/nu10111675.

27. Tulipano G, Vergoni AV, Soldi D, Muller EE, Cocchi D. Characterization of the resistance to the anorectic and endocrine effects of leptin in obesity-prone and obesity-resistant rats fed a high-fat diet. J Endocrinol 2004;183(2):289-98. doi: 10.1677/joe.1.05819.

28. Fruh SM. Obesity: risk factors, complications, and strategies for sustainable long-term weight management. J Am Assoc Nurse Pract. 2017;29(S1):3-14. doi: 10.1002/2327-6924.12510.

29. Minihane AM, Vinoy S, Russell WR, Baka A, Roche HM, Tuohy KM, et al. Low-grade inflammation, diet composition and health: curren research evidence and its translation. Br J Nutr. 2015;114(7):999-1012. doi: $10.1017 /$ S0007114515002093.

30. Itoh K, Moriguchi R, Yamada Y, Fujita M, Yamato T, Oumi M, et al. High saturated fatty acid intake induces insulin secretion by elevating gastric inhibitory polypeptide levels in healthy individuals. Nutr Res. 2014;34(8):653-60. doi: 10.1016/j.nutres.2014.07.013
31. Garcia JL, Francisqueti FV, Ferraz APCR, Ferron AJT, Costa MR, Gregolin CS. High sugar-fat diet induces metabolic-inflammatory disorders independent of obesity development. Food Nutr Sci. 2019;10(6):664-77. doi: 10.4236/fns.2019.106049.

32. Lima-Leopoldo AP, Leopoldo AS, Sugizaki MM, Bruno A, Nascimento AF, Luvizotto RA, et al. Myocardial dysfunction and abnormalities in intracellular calcium handling in obese rats. Arq Bras Cardiol. 2011;97(3):232-40. doi: 10.1590/s0066-782x2011005000061.

33. Dhanasekaran A, Gruenloh SK, Buonaccorsi JN, Zhang R, Gross GJ, Falck JR, et al. Multiple antiapoptotic targets of the PI3K/Akt survival pathway are activated by epoxyeicosatrienoic acids to protect cardiomyocytes from hypoxia/anoxia. Am J Physiol Heart Circ Physiol. 2008;294(2):724-35. doi: 10.1152/ajpheart.00979.2007.

34. Zeidan A, Hunter JC, Javadov S, Karmazyn M. mTOR mediates RhoAdependent leptin-induced cardiomyocyte hypertrophy. Mol Cell Biochem. 2011;352(1-2):99-108. doi: 10.1007/s11010-011-0744-2.

35. Leifheit-Nestler M, Wagner NM, Gogiraju R, Didié M, Konstantinides S, Hasenfuss G, et al. Importance of leptin signaling and signal transducer and activator of transcription-3 activation in mediating the cardiac hypertrophy associated with obesity. J Transl Med. 2013;11:170. doi: 10.1186/1479-5876-11-170.

36. Rider OJ, Francis JM, Ali MK, Byrne J, Clarke K, Neubauer S, et al. Determinants of left ventricular mass in obesity; a cardiovascular magnetic resonance study. J Cardiovasc Magn Reson. 2009;11(1):9. doi: 10.1186/1532-429X-11-9.

37. Francischi RPP, Pereira LO, Freitas CS, Klopfer M, Santos RC, Vieira P, et al. Obesidade: atualização sobre sua etiologia, morbidade e tratamento. Rev Nutr. 2000:13(1):17-28. doi: 10.1590/S1415-52732000000100003.

38. Schram K, Girolamo S, Madani S, Munoz D, Thong F, Sweeney G. Leptin regulates MMP-2, TIMP-1 and collagen synthesis via p38 MAPK in HL-1 murine cardiomyocytes. Cell Mol Biol Lett. 2010;15(4):551-63. doi: 10.2478/s11658-010-0027-z.

39. Madani S, De Girolamo S, Muñoz DM, Li RK, Sweeney G. Direct effects of leptin on size and extracellular matrix components of human pediatric ventricular myocytes. Cardiovasc Res. 2006;69(3):716-25. doi: 10.1016/j. cardiores.2005.11.022

40. Mill JG, Vassallo DV. Hipertrofia cardíaca. Rev Bras Hipertens. 2001;8(1):63-75.

41. Schram K, Ganguly R, No EK, Fang X, Thong FS, Sweeney G. Regulation of MT1-MMP and MMP-2 by leptin in cardiac fibroblasts involves Rho/ROCK-dependent actin cytoskeletal reorganization and leads to enhanced cell migration. Endocrinology. 2011;152(5):2037-47. doi: 10.1210/en.2010-1166).

42. Rababa'h AM, Guillory AN, Mustafa R, Hijjawi T. Oxidative stress and cardiac remodeling: an updated edge. Curr Cardiol Rev. 2018;14(1):53-59. doi: 10.2174/1573403X14666180111145207.

43. Abel ED, Litwin SE, Sweeney G. Cardiac remodeling in obesity. Physiol Rev. 2008;88(2):389-419. doi: 10.1152/physrev.00017.2007. 